\title{
Multiband Charge-Coupled Device
}

\author{
Chu-En Chang, Julie D. Segal, Aaron J. Roodman, Roger T. Howe, Fellow, IEEE, and Christopher J. Kenney
}

\begin{abstract}
We present the multiband charge-coupled device (CCD), a new CCD architecture that contains multiple vertically stacked active layers, capable of generating color images without the use of external filters. One of the applications is to embed the band-resolving capability of astronomical cameras into the CCD and therefore improve the overall survey speed of the telescope. As a proof of concept, we describe a three-layer, three-phase, front-illuminated prototype and simulate its multiband absorption and charge-detection performance.
\end{abstract}

\section{INTRODUCTION}

$\mathrm{T}$ HE charge-coupled device (CCD) has been the imager of choice in astronomy for decades, thanks to its simplicity and the great advances in quality and feature [1]. In state-ofthe-art astronomical telescopes, the CCD is a key component in achieving high light-collecting efficiency, which is crucial to astronomical and cosmological studies [2], [3].

However, because the CCD is monochromatic by nature, the use of color filters is required to obtain color images. Since it is important in astronomy that the imager has nearly $100 \%$ fill factor, the camera is equipped with multiple color filters that are comparable to the focal plane in size. For each field of view, filters are applied sequentially and multiple exposures are taken to form images in different color bands. Since astronomical radiation is wide-band in general, the system quantum efficiency $(\mathrm{QE})$, which we define as the ratio of the number of recorded photons to the total number of incident photons during all exposures for one field of view, is far less than unity. In fact, assuming an ideal imaging system and color filters with approximately equal bandwidths, one can easily see that

$$
\text { System } Q E \leq \frac{\text { Imager } Q E}{\text { Number of filters }},
$$

indicating that the light-collecting efficiency of a telescope is greatly hampered by the use of filters.

This limitation of current imagers provided the motivation for our proposed multiband CCD architecture, which provides for high QE color imaging without the use of sequential filters, so that the light-collecting efficiency and consequently the overall survey speed of a telescope can be improved by multiple times according to (1).

Manuscript received November 16, 2012. SLAC-PUB-15286. This work was supported by the U.S. Department of Energy under Contract No. DEAC02-76-SF00515.

C.-E. Chang and R. T. Howe are with the Department of Electrical Engineering, Leland Stanford Junior University, Stanford, CA 94305 USA (email: cechang@stanford.edu).

J. D. Segal, C. J. Kenney, and A. J. Roodman are with the SLAC National Accelerator Laboratory, Menlo Park, CA 94025 USA.

\section{MultiBAND CCD CONCEPT}

The multiband CCD is fabricated on a silicon-on-insulator (SOI) wafer with multiple silicon device layers. It is similar to the conventional CCD in most senses, except that different layers are designed to interact primarily with photons in different wavelength bands, as shown in Fig. 1(a). This concept is inspired by the fact that the absorptivity and penetration depth of silicon vary greatly with wavelength, as shown in Fig. 1(b). By using the SOI wafer, electron-hole pairs generated at different depths by photons of different wavelengths can be collected by gate potential but still be physically separated in different horizontal active layers by buried silicon dioxide layers, forming vertically stacked charge packets. Subsequently, the stacked packets are transferred together by the same gate voltage but detected at separate sense nodes. The charge from different active layers then forms images in different colors.

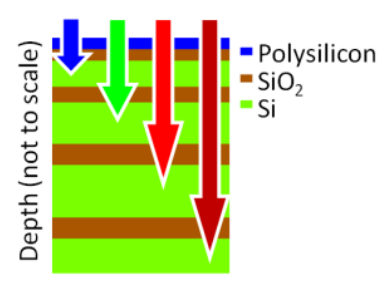

(a)

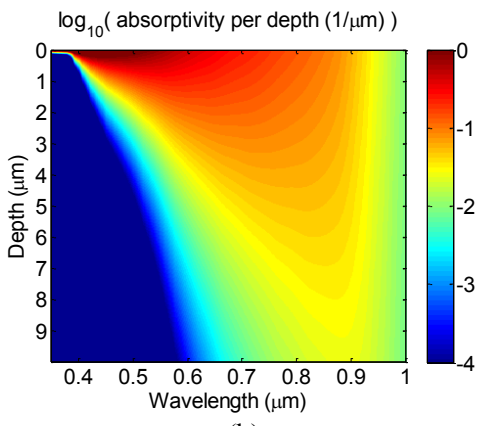

(b)
Fig. 1. (a) Multiband CCD implemented with a multi-layer silicon-oninsulator wafer. Different layers are designed to interact primarily with photons in different wavelength bands, as shown by the arrows. (b) The absorptivity of silicon versus wavelength and depth into the substrate.

The idea of utilizing wavelength-dependent penetration depth to gain spectral information has been previously implemented in two CMOS imagers. The first one contains pixels with an array of contacts that separately collect carriers at different depths [4]. It suffers from variable color crosstalk that depends on excess carrier density because of electric field screening. The second one employs nested triple $p-n$ junctions [5]. Both devices suffer from limited fill factor, because the size of the photosensitive area depends on depth. And since they collect charge with reverse-biased p-n junctions, the vertical dimensions are limited by dopant diffusion and field penetration to less than about $10 \mu \mathrm{m}$, which limits the QE for the astronomically important near-infrared band.

The proposed multiband CCD's color crosstalk is fixed by the thicknesses of the silicon device layers. The pixel size is determined by the size of the gate, as with a conventional CCD. Moreover, the image-enhancement techniques developed for 
astronomical CCDs, such as back illumination and full depletion, can be applied to this new architecture. The number of colors can be increased to six or even more, by simply adding more layers to the SOI wafer, making this architecture attractive for astronomical CCD imagers. In addition, commercial RGB CCD imagers can achieve three-fold improvements in fill factor and consequently system QE by replacing mosaic filters with the multiband $\mathrm{CCD}$ architecture.

\section{PRototype DESIGN}

In order to demonstrate the multiband architecture, we present an initial proof-of-concept design. The structural features are kept simple to expedite design, simulation, and fabrication. The CCD design uses front illumination, polysilicon gates, three-phase pixels, channel-stop trenches, and three active layers for three wavelength bands covering wavelength from $400 \mathrm{~nm}$ to $1000 \mathrm{~nm}$. Additionally, we use ntype silicon substrates.

\section{A. Optical Design}

The scattering-matrix method is used to model the optical response of the 1D structure in Fig. 1, assuming published optical constants [6]. Fig. 2 shows an optimized layer stack and the corresponding absorptivity spectra. These results are encouraging. First, the absorptivity of different active layers is separated into three separate wavelength bands. Second, the overall absorptivity is on par with that of a conventional CCD. In addition, since the system $\mathrm{QE}$ of the multiband $\mathrm{CCD}$ is not bounded by Eq. (1), it can be close to the overall absorptivity if charge transfer and detection are carefully handled. Compared to the conventional CCD with three filters, the new architecture is clearly superior based on system $\mathrm{QE}$, as shown in Fig. 2(a). More importantly, its system QE advantage will grow further as more colors are added, because the conventional CCD's system QE will decrease with each additional color (Eq. (1)).

\section{B. Buried Channel Design}

Buried-channel operation is an important feature that allows the CCDs to achieve high charge transfer efficiency [1]. Since a buried channel is typically formed by an additional doped layer located near the oxide-silicon interface, this technique is compatible with the multiband CCD. However, to simplify the fabrication of the initial device, we choose to optimize the doping profile only for the channel in the middle active layer.

An ion implantation step is designed to penetrate the first two active layers, moving the first two channels away from the gate, as illustrated by the background colors in Fig. 3. The doping profile is found using a commercial process simulator [7]. The potential profile is calculated with a method generalized from [8] and also plotted in Fig. 3. Since the signal carriers are holes, the channels are located at the local potential minima in each silicon layer. Note that the channel of the middle layer is shifted away from adjacent oxide-silicon interfaces, as desired.

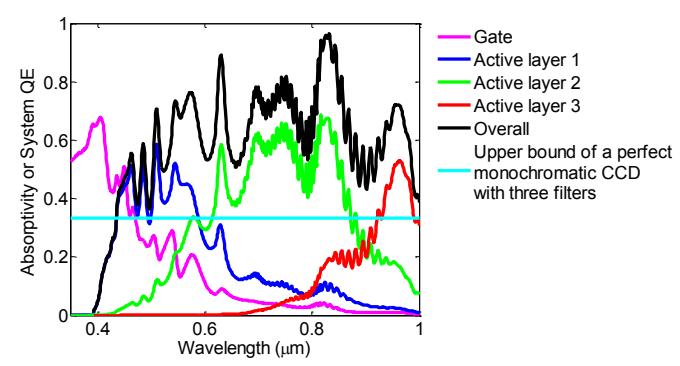

(a) (b)

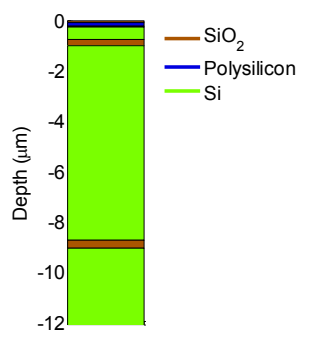

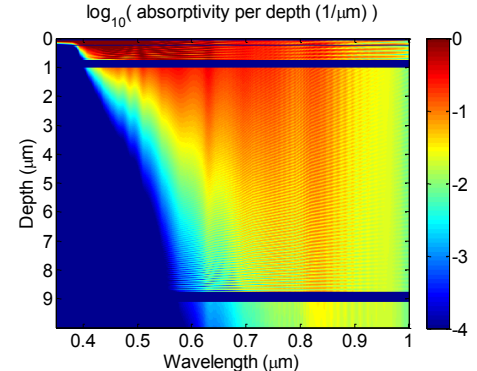

(c)
Fig. 2. Optical performance of the prototype device calculated with the scattering-matrix method. (a) Optimized absorptivity spectra of layers. (b) The 1D device structure. (c) Absorptivity versus wavelength and depth into the substrate. Blue horizontal lines correspond to non-absorptive silicon dioxide layers.

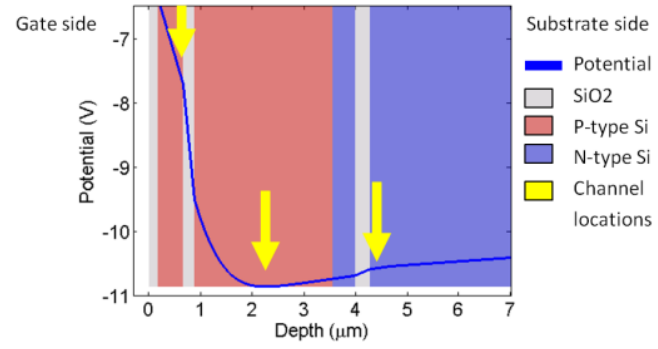

Fig. 3. Buried channel design. The background illustrates the ion implantation from the gate side. The blue curve is the calculated potential profile. Yellow arrows indicate the locations of the channels. The second channel is buried between adjacent oxide-silicon interfaces.

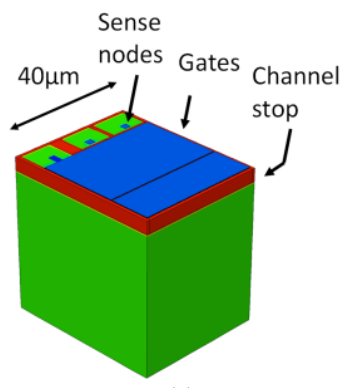

(a)

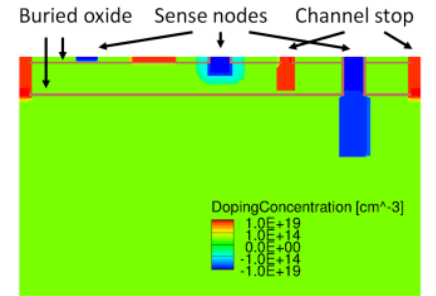

(c)

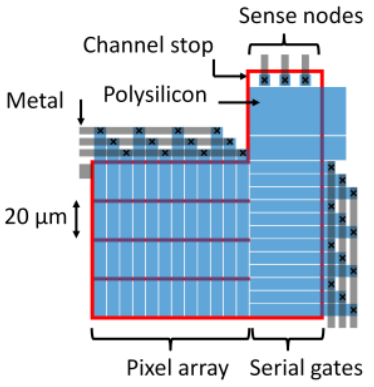

(b)

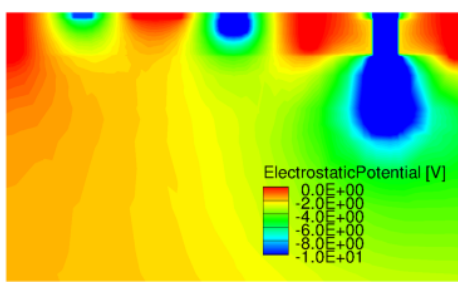

(d)
Fig. 4. Design of sense nodes. (a) 3D TCAD model of the sense node region. (b) Cartoon illustration of the prototype's layout. Note the three sense nodes at the corner. Output amplifier circuits are not shown. (c) A cross section view of the sense nodes. (d) The potential contour at the cross section. 


\section{Sense Nodes}

The design of sense nodes for the multiband CCD is much more involved than for the conventional CCD. Because the output signal is the voltage change at the sense nodes due to incoming charge carriers, any parasitic capacitance between the sense nodes leads to undesirable crosstalk.

In order to explore the design tradeoffs, we used a $3 \mathrm{D}$ TCAD model of the sense node region. Fig. 4 shows an optimized design, which is obtained by heuristically adjusting the geometry and bias voltage to minimize the potential-well overlap of the sense nodes. Since the overlap of the potential wells between two charged metal objects is roughly proportional to the parasitic capacitance, this methodology allows us to effectively minimize crosstalk, while avoiding repeating lengthy simulation of charge detection. Charge detection of the optimized design is simulated only after optimization.

\section{Simulation of Charge Detection}

Charge detection was simulated for the device structure in Fig. 4(a) to verify its performance, using a commercial device simulator [9]. A total of four simulations are presented, all of which begin with biasing the sense nodes, depleting the channels, and forming the potential wells. Once readout starts, simulations begin to differ. In the first, the second, and the third simulations, $1 \times 10^{4}$ excess electron-hole pairs are introduced to simulate optical absorption in the top, the middle, and the bottom layers, respectively. The fourth simulation has no introduced excess charge and serves as the control. Subsequently, the voltage of the sense nodes in each simulation is recorded during readout.

Fig. 5 shows the results of the four simulations. The blue, green, and red curves are at different levels, because the sense nodes are biased at different voltage before readout. The steps at $\mathrm{t}=0.3 \mu \mathrm{s}$ and $0.6 \mu \mathrm{s}$ arise from the switching of the first gate. The experiments start to deviate from the control precisely at $\mathrm{t}$ $=0.6 \mu \mathrm{s}$, because the pixel transfer time is set to $0.6 \mu \mathrm{s}$. The slopes of the solid blue and green curves for $\mathrm{t}>0.6 \mu$ s indicate the pixel transfer time should be increased to several microseconds in order to collect the full signal.

These results are encouraging, because the excess charge in each layer is detected predominantly at the correct sense node with minor crosstalk, which can be mathematically corrected. Formally, we define the charge detection matrix (CDM) as a quantitative measure of how well excess charge is detected:

$$
C D M_{i j}=\frac{V_{i}^{(j)}-V_{i}^{(4)}}{\sum_{i=1}^{3} V_{i}^{(j)}-V_{i}^{(4)}},
$$

where $V_{i}^{(j)}$ is the voltage of the $i^{\text {th }}$ sense node in the $j^{\text {th }}$ simulation, at the completion of the simulation. Table I lists the simulated CDM for the three simulations. Since the diagonal entries are dominant and the matrix is invertible, we find an interesting application of this device: If an unknown monochromatic source is incident on the device, the detected signals at the sense nodes can be used to find the relative percentage of generated carriers in the active layers. The unknown wavelength can then be found from the spectral response shown in Fig. 2(a).

TABLE I. CHARGE DETECTION MATRIX

\begin{tabular}{lccc}
\hline & Simulation 1 & Simulation 2 & Simulation 3 \\
\hline Sense node 1 & $99 \%$ & $7.7 \%$ & $3.9 \%$ \\
Sense node 2 & $0.5 \%$ & $91 \%$ & $15 \%$ \\
Sense node 3 & $0.0 \%$ & $0.8 \%$ & $81 \%$ \\
\hline
\end{tabular}

(a)

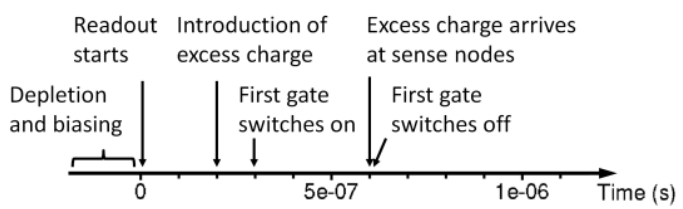

(b)

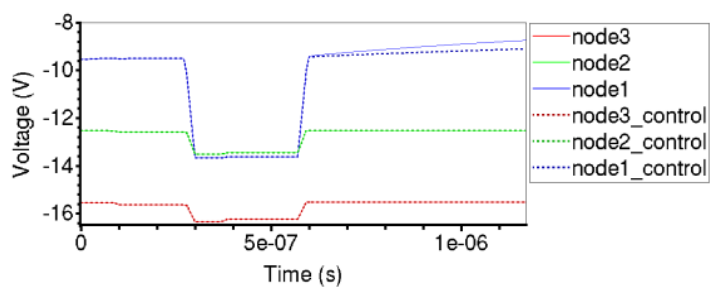

(c)

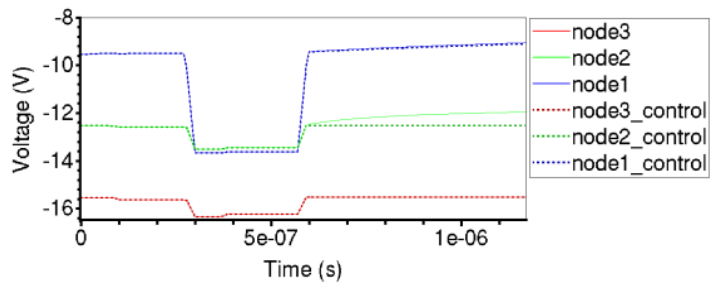

(d)

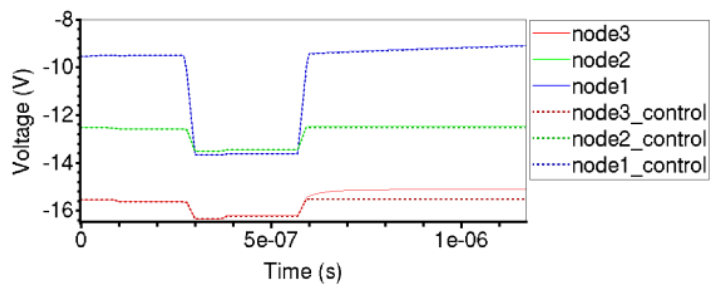

Fig. 5. Simulation of charge detection. Part (a) shows the events in the simulations. Dotted curves are the results of the fourth (control) simulation. Solid curves in (b), (c) and (d) are the results of simulations with excess electron-hole pairs injected into the top, middle, and bottom silicon layer. Blue, green, and red curves indicate the signal voltages at the sense nodes for the top, middle, and bottom silicon layers, respectively.

\section{FABRICATION}

Fabrication of the initial design is underway in the Stanford Nanofabrication Facility. Twelve masks are needed and summarized in Table II. The processing resembles CMOS processing in general, except for the use of multi-layer SOI wafers and the deep trenches in Fig. 4(c). The deep reactive ion etching needed for the trenches is a standard process in MEMS [10]. The process module for the oxide-wrapped sense nodes found in Fig. 4(c) has been developed, as shown in Fig. 6. 


\section{CONCLUSION}

We have presented the multiband CCD concept, a new architecture for color imaging that can greatly improve the system QE of astronomical telescopes. It is extensible to six or more bands, and is also compatible with back illumination and full depletion. A three-layer, three-phase, front-illuminated initial design was simulated, with the results indicating promising performance. Fabrication of the initial design is underway in the Stanford Nanofabrication Facility.

TABLE II. MASKS

\begin{tabular}{cl}
\hline Mask \# & Purpose \\
\hline 1 & Sense node 2 \\
2 & Sense node 3 \\
3 & Channel stop 1 \\
4 & Channel stop 2 \\
5 & Channel stop 3 \\
6 & Buried channel implantation \\
7 & Polysilicon gate \\
8 & Sense node 1 and gate implantation \\
9 & Via \\
10 & Metal 1 \\
11 & Metal 2 \\
12 & Pad opening \\
\hline
\end{tabular}

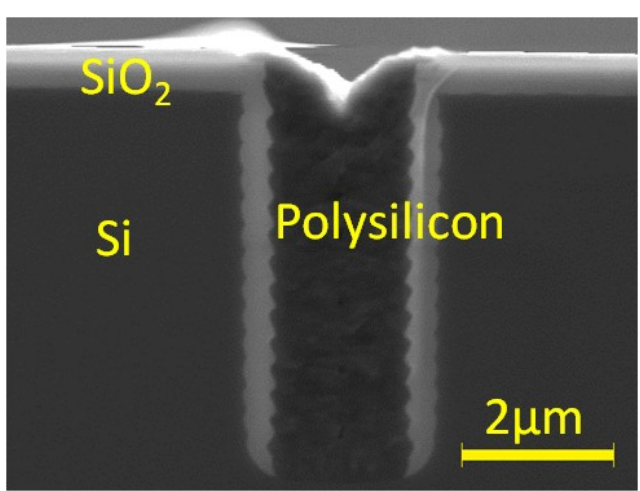

Fig. 6. Scanning electron micrograph of an oxide-wrapped sense node.

\section{REFERENCES}

[1] J. R. Janesick, Scientific Charge-Coupled Devices. Bellingham, WA: SPIE, 2001.

[2] J. A. Tyson and the LSST Collaboration, "Large Synoptic Survey Telescope: overview," Proc. SPIE, vol. 4836, pp. 10-20, 2002.

[3] D. G. York and the SDSS Collaboration, "The Sloan Digital Sky Survey: technical summary," the Astronomical Journal, vol. 120, pp. 1579-1587, Sep. 2000.

[4] G. Langfelder, A. Longoni and F. Zaraga, "Further developments on a novel color sensitive CMOS detector," Proc. SPIE, vol. 7356, Optical Sensors 2009, no. 73562A, May 18, 2009.

[5] R. B. Merrill, "Vertical color filter detector group and array," U.S. Patent 6,632,701 B2, Oct. 14, 2003.

[6] E. D. Palik, Handbook of Optical Constants of Solids. New York: Academic, 1985.

[7] Sentaurus Process User Guide, Version D-2010.03, Synopsys, Inc., Mountain View, CA, 2010.

[8] H. El-Sissi and R. S. C. Cobbold, "One-dimensional study of buriedchannel charge-coupled devices," IEEE Trans. Electron Devices, vol. 21, no. 7, pp. 437- 447, Jul. 1974.

[9] Sentaurus Device User Guide, Version D-2010.03, Synopsys, Inc., Mountain View, CA, 2010.

[10] F. Laermer, "Method of anisotropic etching of silicon," U.S. Patent 6,531,068 B2, Mar. 11, 2003. 